IAU Colloquium 164: Radio Emission from Galactic and Extragalactic Compact Sources

ASP Conference Series, Vol. 144, 1998

J. A. Zensus, G. B. Taylor, \&J.M. Wrobel (eds.)

\title{
The Effects of Light Travel Time on the Appearance of Relativis- tic Jets
}

\author{
A. J. Mioduszewski \\ JIVE/National Radio Astronomy Observatory, Socorro, NM 87801, U.S.A. \\ P. A. Hughes \\ University of Michigan, Ann Arbor, MI 48109-1090, U.S.A. \\ G. C. Duncan \\ Bowling Green State University, Bowling Green, $\mathrm{OH} 43403$, U.S.A.
}

\begin{abstract}
We present simulated maps showing the appearance in total intensity of flows computed using a relativistic hydrodynamic code (Duncan \& Hughes 1994: ApJ, 436, L119). The radiation transfer calculations allow for full treatment of relativistic effects, such as Doppler boosting and time delay. Depending on viewing angle, and the speed of emitting features, the appearance of a jet can be strongly influenced by either effect; in both cases the map differs dramatically from the morphology exhibited by the hydrodynamic quantities.
\end{abstract}

\section{Introduction}

Many properties of active galactic nuclei (AGN) have been attributed to relativistic effects. Doppler boosting and light travel time effects can influence many properties of $\mathrm{AGN}$, if bulk relativistic motion and viewing angles close to the line of sight $\left(\sim 0^{\circ}-30^{\circ}\right)$ are assumed. Doppler boosting explains hot spots and the absence of counterjets; and light travel time effects explain the rapid variability and superluminal motion. To study the influence of relativistic effects on the appearance of jets, we have computed the emitted synchrotron flux (i.e. simulated "maps") of the relativistic hydrodynamical simulations of Duncan \& Hughes (1994). We have taken two approaches to mapping these simulations: including Doppler boosting only and full treatment of relativistic effects (i.e., including both Doppler boosting and light travel time effects). Here we present the results of these studies, highlighting the dramatic change in the appearance of jets when a full treatment of relativistic effects is included.

\section{The Simulated Maps}

The synchrotron radiation transfer calculations for the total intensity were performed as described in Mioduszewski, Hughes, \& Duncan (1997). Figure 1 shows select frames of the evolution of relativistic hydrodynamical simulation and flux intensity of the jet at $30^{\circ}$. Notice that there is a one-to-one correspondence between the features in the hydrodynamic simulations and Doppler boost-only maps but not true for the fully relativistic maps. Since light travel time is included in the fully relativistic maps, features in the-maps can arise from different times during the evolution of the jet. Individual features of the fully relativistic maps were studied to discover their origin. One surprising result is that the two features designated by arrows in the fourth panel from the top of Fig 1c. arise from the same shock at different epochs along the total evolution of the jet. 


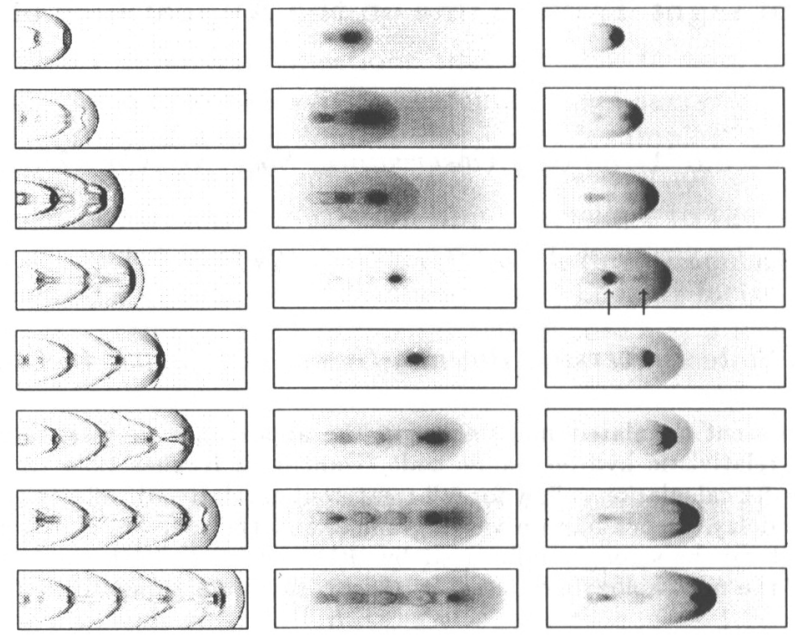

Figure 1. a. Images of laboratory frame pressure gradient at, starting from the top, frames which are $26 \%, 32 \%, 43 \%, 55 \%, 66 \%, 77 \%, 88 \%$ and $100 \%$ along the total run time of the hydrodynamical simulation. This simulation has an adiabatic index of $4 / 3$, the injection Lorentz factor was sinusoidally modulated between $\sim 1$ and 10 to induce perturbations and the density of the ambient medium is 10 times the density inside the jet. The darker grey, the higher the pressure gradient. b. Maps of the intensity distribution for Doppler boost only and c. full relativistic effects.

\section{Conclusions}

The inclusion of light travel time effects: 1) induces relativistic aberration which has a considerable effect on the appearance of the jet components; 2) causes many of the shocks/features that appear in the hydrodynamical simulation to not appear in the maps; 3 ) produces other counter-intuitive phenomena such as a single shock causing spatially separated features in a map. In other words, the underlying jet and the radio map may have structures that are very different. An important conclusion of this work is that simple interpretations which depend a direct connection between the high resolution maps of jets and the underlying flow are not valid.

Acknowledgments. This work was supported by NSF grant AST 9120224, by the Ohio Supercomputer Center from a Cray Research Software Development Grant and by the European Union under contract CHGECT920011. The National Radio Astronomy Observatory is a facility of the National Science Foundation, operated under a cooperative agreement by Associated Universities, Inc.

\section{References}

Duncan, G. C., \& Hughes, P. A. 1994. ApJ, 436, L119-122.

Mioduszewski, A. J., Hughes, P. A., \& Duncan, G. C. 1997. ApJ, 476, 649-665. 